\title{
Original
}

\section{The Role of the Posterior Oblique Ligament in Tears of the Medial Collateral Ligament of the Knee}

\author{
Hiroshi Higuchi ${ }^{1}$, Atsushi Kobayashi ${ }^{1}$, Keiko Ikeda ${ }^{1}$ and Kazuhisa Hatayama ${ }^{2}$ \\ 1 Department of Orthopaedic Surgery, Asakura Sports Rehabilitation Clinic, 249-1 Asakura-machi, Maebashi, Gunma 371-0811, Japan \\ 2 Department of Orthopaedic Surgery, Gunma Chuo General Hospital, 1-7-13 Kou-un-cho, Maebashi, Gunma 371-0025, Japan
}

\begin{abstract}
Purpose: To evaluate a novel method to diagnose injury of the postero-oblique ligament (POL) complicating injury of the medial collateral ligament (MCL) of the knee and its treatment.

Methods: Five POL-injured patients with grade III MCL injury were enrolled. Injury of the POL was diagnosed by valgus stress radiography with an external rotational force applied to the leg at 30r knee flexion (VSRE) and arthroscopy.

Results: On VSRE, in the neutral position widening of the medial joint space of the knee compared to the intact side was seen in all patients. Arthroscopy also showed marked widening of the joint space at the MCL and posteriorly to it. Intraoperatively, instability on the posteromedial side of the knee remained following the repair of the MCL alone, but stability was obtained by repair of the POL. No instability of the knee was noted on VSRE even two years postoperatively, and the patients could return to their former sport activities.

Conclusions: VSRE was effective for diagnosing injury of the POL. If the POL-injury is detected using this screening test, repair of the POL as well as MCL would be considered necessary.
\end{abstract}

\begin{tabular}{l} 
Article Information \\
\hline Key words: \\
Posterior Oblique Ligament, \\
Knee, \\
Stress Radiography, \\
Arthroscopy \\
\hline Publication history: \\
Received: April 17, 2017 \\
Revised: June 6, 2017 \\
Accepted: June 15, 2017 \\
\hline Corresponding author: \\
Hiroshi Higuchi \\
Department of Orthopaedic Surgery, Asakura Sports Reha- \\
bilitation Clinic, 249-1 Asakura-machi, Maebashi, Gunma \\
371-0811, Japan \\
Tel: +81-272-65-6522 \\
E-mail: jaguar156z@ yahoo.co.jp \\
\hline
\end{tabular}

\section{Introduction}

This study was initiated on the basis of our experience in treating patients with old postero-oblique ligament (POL) injury. A 21-year-old soccer player was tackled and injured, as his right knee was extroverted and externally rotated at about 30 knee flexion. $\mathrm{He}$ consulted another clinic, was diagnosed with medial collateral ligament (MCL) injury, and treated conservatively including knee bracing. As a result, he recovered until he became able to play soccer again within 4 months. However, as the feeling of dislocation at the external rotation during the cutting maneuver in about $45^{\circ}$ flexion of the right knee persisted, he consulted our department for the first time 6 months after the injury. Physical findings included no instability of the MCL and negative manual valgus stress tests on both $0^{\circ}$ and $30^{\circ}$ knee flexion. Initially, we did not understand the patient's complaint. However, as the complaint continued, we examined the knee instability by having the patient perform the cutting maneuver under fluoroscopy. As a result, we confirmed widening of the medial joint space during extroversion of the right knee on about $40^{\circ}$ flexion combined with external rotation of the leg. Reviewing past reports, ${ }^{1-4}$ we judged the symptom to have been caused by POL injury accompanied by antero-medial rotatory instability (AMRI). On the basis of the experience with this patient, we re-evaluated the diagnostic method for POL injury.

To diagnose POL injury, we devised valgus stress radiography on $30^{\circ} \mathrm{knee}$ flexion instead of valgus stress radiography in knee extension, which is performed for 
the diagnosis of MCL injury. First, valgus stress radiography is performed with the leg in the neutral position. Because of MCL injury, the medial joint space is wider in the injured limb compared with the intact side (Fig. 1). Next, stress radiography is performed similarly by applying an external rotational force to the leg. In patients with POL injury, the medial joint space is widened more markedly when the crus is externally rotated than the leg in the neutral position (Fig. 2). In patients with grade III MCL injury not accompanied by POL injury, no change is observed on valgus stress radiographic findings during external rotation. By screening patients with grade III MCL injury employing this method, we encountered four more patients with POL injury accompanied by AMRI. We evaluated the function of the POL in MCL injury on the basis of the experience with these five patients.
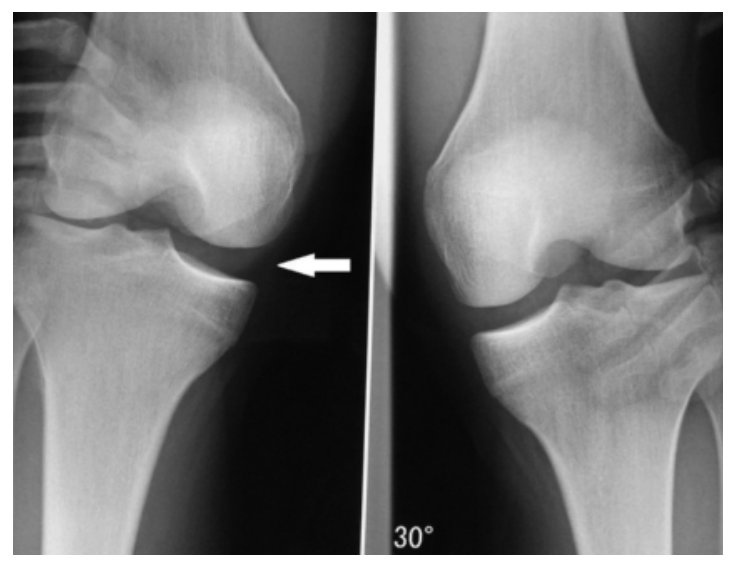

Fig. 1 Valgus stress radiography on $30^{\circ}$ knee flexion is performed with the leg in the neutral position. Right knee is injured (Arrow head).
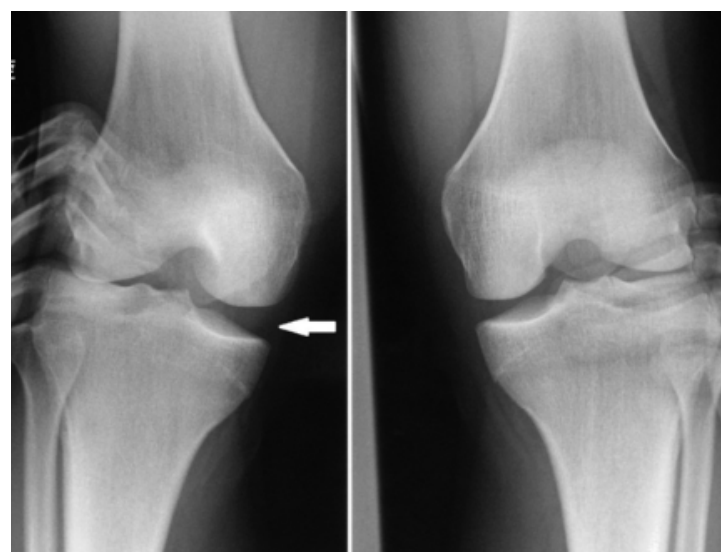

Fig. 2 Valgus stress radiography on $30^{\circ}$ knee flexion applying an external rotational force showed that the medial joint space is widened more markedly than the leg in the neutral position. Right knee is injured (Arrow head).

\section{Materials and Methods}

After we encountered the first patient, we screened patients with grade III MCL injury who visited our clinic by valgus stress radiography on 30 knee flexion applying an external rotational force, and diagnosed POL injury in four more patients. The five patients consisted of three with injury of the MCL alone and two with combined injury of the ACL +MCL. All patients showed a positive valgus stress test on $30^{\circ}$ knee flexion and more marked widening of the medial joint space on the test with an external rotational force. They also showed a positive anterior drawer test on $90^{\circ}$ knee flexion. On MRI, high-signal changes were noted in the superficial and deep layers of the MCL in all patients, allowing the diagnosis of rupture. However, there was no characteristic MR finding that permitted the identification of the injury type of the POL, which is located posteriorly to the MCL. Among other tests, arthroscopy was particularly effective. On arthroscopy of the medial joint space at 90 knee flexion by vertical hanging of the lower limb, easy and clear visibility of the posterior segment of the medial meniscus (MM), which is normally hidden behind the internal condyle of the femur and difficult to observe, is a characteristic finding due to widening of the medial joint space of the knee caused by loosening of the articular capsule and ligaments posterior to the MCL. When the leg is also extroverted from the same limb position, the articular capsule posterior to the MCL opens like a shellfish opening its shell, making the looseness of the posteromedial parts of the joint more notable. This phenomenon, observed in all patients, was named the "shellfish opening sign" (Fig. 3).

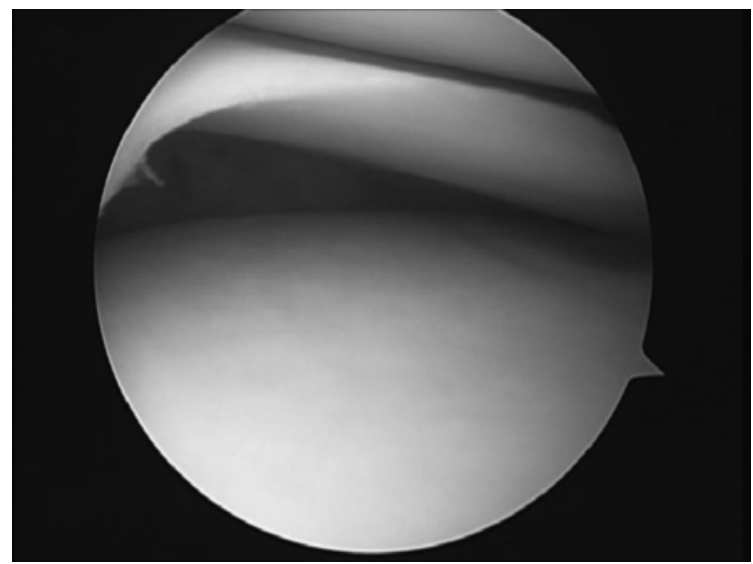

Fig. 3 Widening of the medial joint space of the knee caused by loosening of the articular capsule and ligaments posterior to the medial collateral ligament under arthroscopic examination by vertical hanging of the lower limb. Applying external rotation, medial meniscus showed markedly instability that was named the "shellfish opening sign”.

Surgical technique: In all patients, the POL was repaired by suturing using a Panalok Anchor (Depuy Mitek Inc, Raynham, MA). The posterior part of the knee was exposed from the medial side, and the $\mathrm{S}$ MCL and POL were identified (Fig. 4). When an external force was applied on 30 knee flexion, the gap of the ruptured MCL widened along the course of the ligament fibers, and the gap of the ruptured POL posterior to the MCL was widened and rotated. First, the MCL was repaired by suturing, and the valgus 


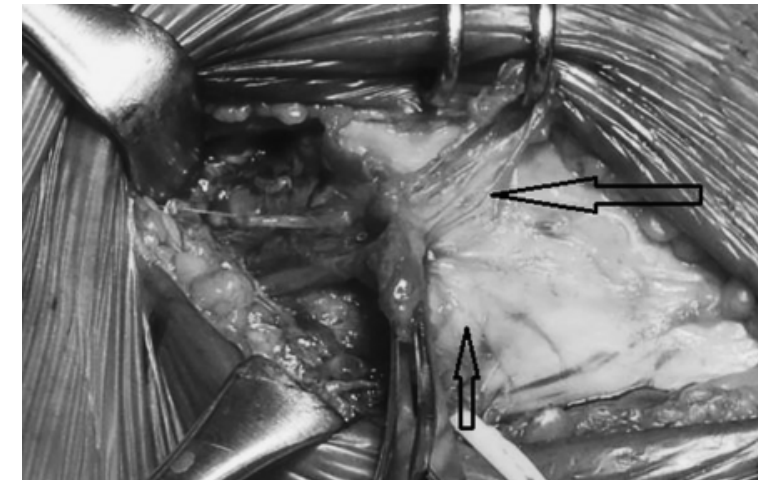

Fig. 4 The posterior part of the knee was exposed from the medial side, and the superficial-MCL (Bigger arrow head) and POL (Smaller arrow head) were identified. After releasing both ligaments from the medial epicondyle, pull-tension was applied to the each ligament. It confirmed that each ligament had tonus enough after pulling ligament.

stress test of the knee on $30^{\circ}$ flexion was performed again. While the MCL was stabilized by suturing, rotational instability remained at the rupture site of the POL posteriorly to the MCL. Thus, we learned that AMRI associated with POL injury cannot be controlled by repair of the MCL alone. The POL was repaired by suturing according to the injury type. In patients with loosening of the POL due to its detachment from the medial epicondyle of the femur, this area was lifted up using a Panalok Anchor and sutured (Fig 5). Also, in patients with rupture of the superficial part, rotational instability could be controlled by suturing the ruptured ends and then suturing the superficial part and S-MCL by partially overlapping them.

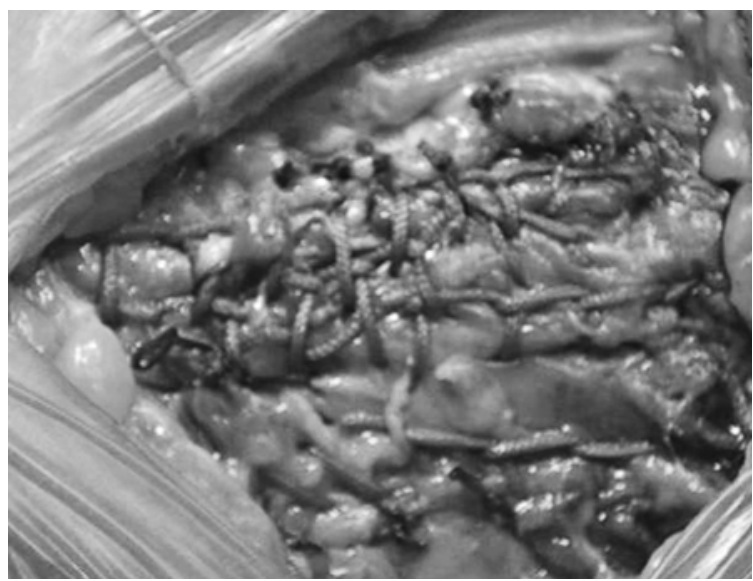

Fig. 5 After applying Krakow suture to both the MCL and the POL, each ligament was advanced to the central side. After having confirmed that each ligament had tonus enough, both ligaments were fixed using suture anchors.

\section{Results}

Each patient was followed-up for two year after surgery. All patients could return to their former sport activities and showed a normal manual valgus stress test. Also, there was no restriction in the knee range of motion, and no difference was noted in the findings on valgus stress radiography on 30 knee flexion with an external rotational force to the leg between the injured and intact limbs. In addition, arthroscopy was performed one year after surgery in all patients. The "shellfish opening sign" had disappeared, and the posterior segment of the MM was hidden behind the internal condyle of the femur (Fig 6). Loosening of the joint space posterior to the MCL also disappeared after the repair of the POL.

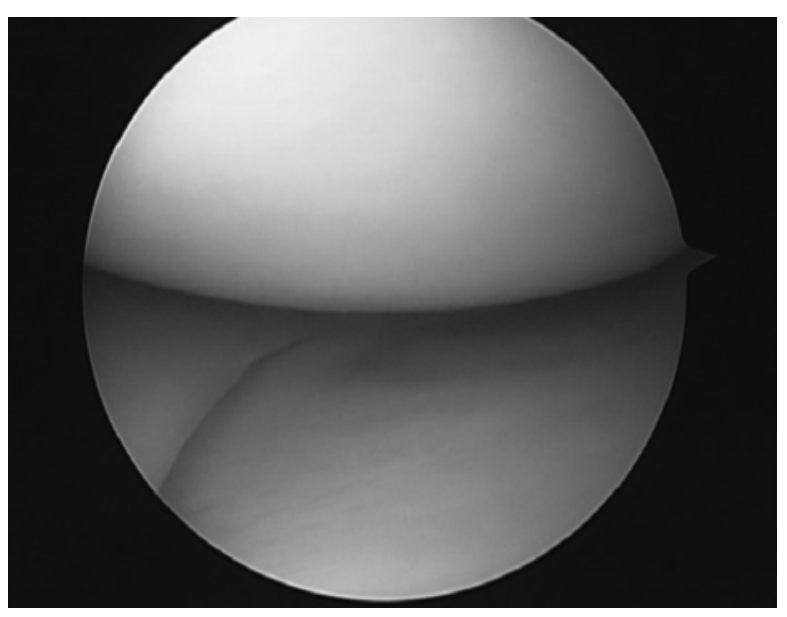

Fig. 6 Postoperative arthroscopy showed that the posterior segment of the MM was hidden behind the internal condyle of the femur. The "shellfish opening sign" was disappeared.

\section{Discussion}

There have been a few basic studies on the anatomical role of the POL. ${ }^{5-9}$ The POL, defined by Hughston and Eilers, ${ }^{5}$ is located posteriorly to the S-MCL and occupies $1 / 3$ of the posteromedial part of the knee. It is divided into 3 parts, i.e., capsular, tibial, and superficial, each of which proximally transitions into the semimembranosus tendon. They speculated that, if injury of the MCL is complicated by that of the POL, restoration of the medial stability would not be expected without POL repair. ${ }^{5}$ Also, as the POL is closely related to the MM, they stabilized the MM and simultaneously pulled the MM posteriorly on flexion of the knee, so that it would not be caught by the joint space. $^{7}$

Nonsurgical treatments are often selected for MCL injuries, and their outcomes are favorable. ${ }^{10-12}$ However, some patients with grade III MCL injury exhibit AMRI, and instability may persist after conservative treatment. Also, how the MCL injury should be treated in complex ligament injuries such as ACL+ MCL injury remains controversial, ${ }^{11-13}$ and there is no standard as to which MCL injuries require surgical treatment. From the experience in treating patients with chronic POL injury, valgus stress radiography in $30^{\circ}$ knee flexion with an external rotational force applied to the leg was shown to be effective for the diagnosis of POL injury in patients with grade III MCL injury. If POL injury has been detected by screening using this technique, surgical repair of the 
POL as well as the MCL is considered necessary, because intraoperative findings showed that posteromedial knee instability cannot be controlled by the repair of the MCL alone. Also, if the "shellfish opening sign", the widening of the medial joint space of the knee due to loosening of the articular capsule and ligaments posterior to the MCL, is noted by arthroscopy, open surgery should be evaluated in consideration of POL injury. We often encounter MCL injuries in daily orthopedic practice, but caution is needed as the prognosis of grade III MCL injury differs depending on whether it is complicated by POL injury.

\section{Conclusions}

Valgus stress radiography in $30^{\circ}$ knee flexion with an external rotational force applied to the leg is effective for the diagnosis of POL injury in patients with grade III MCL injury. Also, arthroscopy of the medial joint space with the leg both hanging vertically and applying external rotational force showed markedly widening of the joint space at the MCL and posteriorly to it. If POL injury has been revealed by screening using these tests, repair of the POL as well as the MCL would be considered necessary.

\section{References}

1. Slocum DB, Larson RL. Rotatory instability of the knee. Its pathogenesis and a clinical test to demonstrate its presence. J Bone Joint Surg Am 1968; 50: 211-225.

2. Hughston JC, Andrews JR, Cross MJ, et al. Classification of knee ligament instabilities. Part I. The medial compartment and cruciate ligaments. J Bone Joint Surg Am 1976; 58: $159-172$.

3. Hughston JC, Barrett GR. Acute anteromedial rotatory instability. Long-term results of surgical repair. J Bone Joint Surg Am 1983; 65: 145-153.

4. Kurimura M, Matsumoto H, Fujikawa K, et al. Factors for the presence of anteromedial rotatory instability of the knee. J Orthop Sci 2004; 9: 380-385.

5. Hughston JC, Eilers AF. The role of the posterior oblique ligament in repairs of acute medial (collateral) ligament tears of the knee. J Bone Joint Surg Am 1973; 55: 923-940.

6. Fischer RA, Arms SW, Johnson RJ, et al. The functional relationship of the posterior oblique ligament to the medial collateral ligament of the human knee. Am J Sports Med 1985; 13: 390-397.

7. Sims WF, Jacobson KE. The posteromedial corner of the knee: medial-sided injury patterns revisited. Am J Sports Med 2004; 32: 337-345.

8. Griffith CJ, LaPrade RF, Johansen S, et al. Medial knee injury: Part 1, static function of the individual components of the main medial knee structures. Am J Sports Med 2009; 37: 1762-1770

9. Wijdicks CA, Ewart DT, Nuckley DJ, et al. Structural properties of the primary medial knee ligaments. Am J Sports Med 2010; 38: 1638-1646.

10. Jones RE, Henley MB, Francis P. Nonoperative management of isolated grade III collateral ligament injury in high school football players. Clin Orthop Relat Res 1986: 137140 .

11. Jacobson KE, Chi FS. Evaluation and treatment of medial collateral ligament and medial-sided injuries of the knee. Sports Med Arthrosc 2006; 14: 58-66.

12. Miyamoto RG, Bosco JA, Sherman OH. Treatment of medial collateral ligament injuries. J Am Acad Orthop Surg 2009; 17: 152-161.

13. Sherman MF. The importance of the posterior oblique ligament in repairs of acute tears of the medial ligaments in knees with and without an associated rupture of the anterior cruciate ligament. Results of long-term follow-up. J Bone Joint Surg Am 1995; 77: 969-970. 